\title{
A 3,5-YEAR-OLD GIRL WITH LIFE-THREATENING IDIOPATHIC SYSTEMIC CAPILLARY LEAK SYNDROME DUE TO INFLUENZA B - A CASE REPORT
}

Stavrou Vasillios, Poulos Panagiotis, Christakou Eleni, Straka Kalliopi, Paraschou Dimitra, Kalampalikis Panagiotis, Barbaresou Chariklia

Pediatric Intensive Care Unit, Aghia Sophia Children's Hospital, Athens, Greece

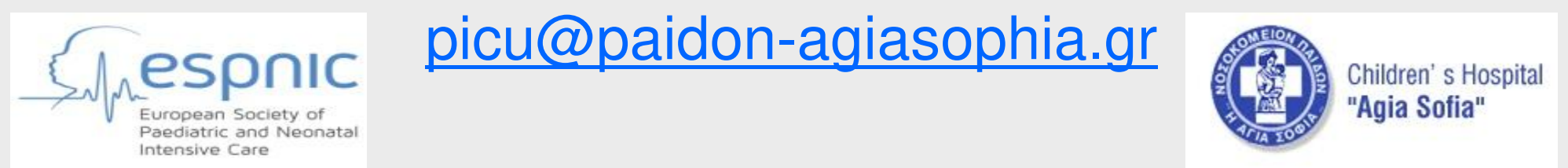

\section{BACKGROUND}

Idiopathic Systemic Capillary Leak Syndrome (ISCLS) is a very rare but life-threatening condition characterized by recurrent episodes of severe hypotension, hypoalbuminemia and marked hemoconcentration, due to plasma leakage to the extravascular compartment. Reports on paediatrics are very few and are usually precipitated by an infectious trigger.

\section{METHODS}

We present a 3,5 year-old girl with ISCLS admitted to PICU with the presumed diagnosis of hypovolemic shock.

\section{RESULTS}

The previously healthy patient presented with a history of fever, cough, vomiting, myalgia and oliguria. On admission to PICU she had signs of poor perfusion, refractory hypotension and reduced level of consciousness. Initial blood tests revealed significant hemoconcentration ( $\mathrm{Hgb} 20,5 \mathrm{~g} / \mathrm{L}$ ),

severe hypoalbuminemia ( $2 \mathrm{~g} / \mathrm{dL}$ ), low sodium and metabolic acidosis. She was resuscitated requiring large amounts of crystalloid fluids and albumin and she was started on antibiotics and oseltamivir. Although infectious workup for presumed sepsis (blood, urine and celebrospinal fluid cultures) failed to reveal a source, nasopharyngeal aspirate tested positive for influenza B virus. On

day 2 her clinical course was complicated by anasarca and weight gain $(+3,5 \mathrm{~kg})$ resulting in compartment syndrome of the right lower extremity and rhabdomyolysis without pulmonary oedema.

Based on the classic triad of hypotension, hypoalbuminemia and hemoconcentration SCLS was diagnosed. She was treated with iv albumin and furosemide which led to eventual recovery. She was discharged a few days later on prophylactic therapy (IVIG and salbutamole p.os).

\section{CONCLUSIONS}

ISCLS should be considered in the differential diagnosis of unexplained hypovolemic shock followed by generalized edema in the resuscitation phase. Accordingly appropriate fluid management is most important. The cause of the marked shift of plasma has not been elucidated although disruption of endothelial junctions, cell retraction and increase of VEGF and angiopoietin-2 have been suggested

\section{SOURCES}

1. Hsu P, Xie Z, Frith K, et al. Idiopathic Systemic Capillary Leak Syndrome in Children. Pediatrics. 2015;135(3):e730-e735.

2. Gousseff M, Arnaud L, Lambert M, Hot A, Hamidou M, Duhaut P, et al. The Systemic Capillary Leak Syndrome: A Case Series of 28 Patients From a European 